\title{
A New Approach for Solving Fully Fuzzy Linear Systems
}

\author{
Amit Kumar, ${ }^{1}$ Neetu, ${ }^{1}$ and Abhinav Bansal ${ }^{2}$ \\ ${ }^{1}$ School of Mathematics and Computer Applications, Thapar University, Patiala 147004, India \\ ${ }^{2}$ Computer Science and Engineering Department, Thapar University, Patiala 147004, India
}

Correspondence should be addressed to Neetu Babbar, neetu_babbar83@rediffmail.com

Received 8 December 2010; Revised 3 March 2011; Accepted 1 April 2011

Academic Editor: Ajith Abraham

Copyright () 2011 Amit Kumar et al. This is an open access article distributed under the Creative Commons Attribution License, which permits unrestricted use, distribution, and reproduction in any medium, provided the original work is properly cited.

\begin{abstract}
Several authors have proposed different methods to find the solution of fully fuzzy linear systems (FFLSs) that is, fuzzy linear system with fuzzy coefficients involving fuzzy variables. But all the existing methods are based on the assumption that all the fuzzy coefficients and the fuzzy variables are nonnegative fuzzy numbers. In this paper a new method is proposed to solve an FFLS with arbitrary coefficients and arbitrary solution vector, that is, there is no restriction on the elements that have been used in the FFLS. The primary objective of this paper is thus to introduce the concept and a computational method for solving FFLS with no non negative constraint on the parameters. The method incorporates the principles of linear programming in solving an FFLS with arbitrary coefficients and is not only easier to understand but also widens the scope of fuzzy linear equations in scientific applications. To show the advantages of the proposed method over existing methods we solve three FFLSs.
\end{abstract}

\section{Introduction}

One field of applied mathematics that has many applications in various areas of science is solving a system of linear equations. Systems of simultaneous linear equations play a major role in various areas such as operational research, physics, statistics, engineering, and social sciences. When the estimation of the system coefficients is imprecise and only some vague knowledge about the actual values of the parameters is available, it may be convenient to represent some or all of them with fuzzy numbers [1]. Fuzzy number arithmetic is widely applied and useful in computation of linear system whose parameters are represented by fuzzy numbers, which are called fuzzy linear systems (FLSs). Buckley and $\mathrm{Qu}$ [2] defined the concept of solving fuzzy equations and their work has been influential in the study of fuzzy linear systems. A general model for solving an $n \times n$ fuzzy linear system whose coefficient matrix is crisp and the right-hand side column is an arbitrary fuzzy vector was first proposed by Friedman et al. [3, 4]. Later some numerical methods to solve similar systems were proposed [5] and extended methods like successive overrelaxation [6] adomian decomposition [7] were also presented. Abbasbandy et al. also described LU decomposition method [8], Conjugate Gradient method [9], and Steepest descent method [10] for solving such system of fuzzy equations. Some iterative methods to solve an FLS were also extended in [11]. The condition of crispness of the coefficient matrix makes all these methods restricted with negligible applications.

In addition, another important kind of fuzzy linear systems are the fully fuzzy linear systems (FFLS) in which all the parameters are fuzzy numbers. Dehghan and Hashemi $[12,13]$ proposed the Adomian decomposition method, and other iterative methods to find the positive fuzzy vector solution of $n \times n$ fully fuzzy linear system. Dehghan et al. [14] proposed some computational methods such as Cramer's rule, Gauss elimination method, LU decomposition method and linear programming approach for finding the approximated solution of FFLS. Muzzioli and Reynaerts [15] introduced an algorithm to find vector solution by transforming the system $A_{1} x+b_{1}=A_{2} x+b_{2}$ into the FFLS $A x=b$ where $A=A_{1}-A_{2}$ and $b=b_{2}-b_{1}$.

Mosleh et al. [16] proposed a method to find the solution of fully fuzzy linear system of the form $A x+b=C x+d$ 
with $A, C$ being square matrices of fuzzy coefficients and $b, d$ being fuzzy number vectors, and the unknown vector $x$ is vector consisting of $n$ fuzzy numbers. Nasseri et al. [17] used certain decomposition methods of the coefficient matrix for solving fully fuzzy linear system of equations. Allahviranloo et al. [18] proposed a numerical method for solving FFLS $A x=b$, when coefficient matrix is positive. Gao [19] proposed a unified iterative scheme for solving nonsquare FFLS with nonnegative constraints.

Nasseri et al. [20] proposed Greville's method to find the positive solution of FFLS. Mosleh et al. [21] discussed symmetric times triangular (ST) decomposition to find the positive solution of FFLS. Nasseri and Zahmatkesh [22] proposed a new method for computing the nonnegative solution of FFLS. Liu [23] developed a block homotopy perturbation method (HPM) for finding the approximation of the solution of FFLS. Nasseri and Sohrabi [24] used a certain decomposition of the coefficient matrix of the FFLS to construct a new algorithm for solving FFLS. Kumar et al. [25] proposed a new method to find the approximate solution of FFLS with trapezoidal fuzzy numbers as parameters. Recently some other methods like homotopy perturbation, splitting methods and other numerical methods have been proposed in [26-29] but possess similar restrictions for solving fuzzy systems. It may be seen that the methods assume the nonnegativity of the coefficients.

In this paper, a new method based upon the decomposition of an FFLS into a nonlinear system and subsequently a linear programming problem, is proposed to find the solution of FFLS without any restriction on coefficient and fuzzy variables. To illustrate the proposed method numerical examples are solved. The rest of paper is organized as follows. In Section 2, some basic definitions and arithmetic operations of triangular fuzzy numbers are reviewed. In Section 3, limitations of the existing methods to solve FFLS are described. In Section 4, a new computational method for solving FFLS with arbitrary coefficient matrix and arbitrary fuzzy variable is introduced. In Section 5, to exemplify the method three FFLSs are solved. The paper is concluded in Section 6.

\section{Preliminaries}

2.1. Basic Definitions. In this section some basic definitions of fuzzy set theory are reviewed $[30,31]$.

Definition 1. A fuzzy number $\tilde{A}$ is said to be nonnegative fuzzy number if the domain of its membership function is a set of nonnegative real numbers $\left(R^{+}\right)$, that is, $\mu_{\widetilde{A}}$ : $R^{+} \rightarrow[0,1]$. The set of nonnegative fuzzy numbers may be represented by $F\left(R^{+}\right)$.

Definition 2. A fuzzy number $\tilde{A}$ is said to be unrestricted fuzzy number if the domain of its membership function is a set of real numbers $(R)$, that is, $\mu_{\tilde{A}}: R \rightarrow[0,1]$. The set of unrestricted fuzzy numbers may be represented by $F(R)$.
Definition 3. A fuzzy number $\tilde{A}=(a, b, c)$ is said to be a triangular fuzzy number if its membership function is given by

$$
\mu_{\widetilde{A}}(x)= \begin{cases}0, & x<a, \\ \frac{x-a}{b-a}, & a \leq x \leq b, \\ \frac{c-x}{c-b}, & b<x \leq c, \\ 0, & x>c,\end{cases}
$$

where $a, b, c \in R$.

Definition 4. A triangular fuzzy number; $\tilde{A}=(a, b, c)$ is said to be unrestricted triangular fuzzy number if $a, b, c \in R$.

Definition 5. A triangular fuzzy number $\tilde{A}=(a, b, c)$ is said to be nonnegative triangular fuzzy number; that is, $\tilde{A} \geq 0$, that is, if and only if $a \geq 0$.

Definition 6. A triangular fuzzy number $\tilde{A}=(a, b, c)$ is said to be zero triangular fuzzy number if and only if $a=0, b=0$, and $c=0$.

Definition 7. Two triangular fuzzy numbers $\widetilde{A}_{1}=(a, b, c)$ and $\tilde{A}_{2}=(e, f, g)$ are said to be equal; that is, $\tilde{A}_{1}=\tilde{A}_{2}$ if and only if $a=e, b=f$, and $c=g$.

Definition 8. A matrix $\tilde{A}=\left(\tilde{a}_{i j}\right)$ is called a fuzzy matrix, if each element of $\tilde{A}$ is a fuzzy number. $\tilde{A}$ will be positive (negative) and denoted by $\tilde{A}>0(\tilde{A}<0)$ if each element of $\tilde{A}$ positive (negative). $\tilde{A}$ will nonpositive (nonnegative) and denoted by $\tilde{A} \leq 0(\tilde{A} \geq 0)$ if each element of $\tilde{A}$ be nonpositive (nonnegative). We may represent $n \times m$ fuzzy matrix $\tilde{A}=\left(\tilde{a}_{i j}\right)_{n \times m}$ where $\tilde{a}_{i j}=\left(a_{i j}, b_{i j}, c_{i j}\right)$.

Definition 9. Let $\widetilde{A}=\left(\tilde{a}_{i j}\right)$ and $\widetilde{B}=\left(\tilde{b}_{i j}\right)$ be two $m \times n$ and $n \times p$ fuzzy matrices. We define $\widetilde{A} \otimes \widetilde{B}=\widetilde{C}=\left(\widetilde{c}_{i j}\right)$ which is the $m \times p$ matrix where $\tilde{c}_{i j}=\sum_{k=1, \ldots, n}^{\oplus} \tilde{a}_{i k} \otimes \tilde{b}_{k j}$.

2.2. Arithmetic Operations on Triangular Fuzzy Numbers. In this subsection addition and multiplication operations between two triangular fuzzy numbers are reviewed [30, 31]. Let $\widetilde{A}_{1}=(a, b, c)$ and $\widetilde{A}_{2}=(e, f, g)$ be two triangular fuzzy numbers; then one has the following.

(i) $\widetilde{A}_{1} \oplus \widetilde{A}_{2}=(a, b, c) \oplus(e, f, g)=(a+e, b+f, c+g)$

(ii) $\widetilde{A}_{1} \ominus \widetilde{A}_{2}=(a, b, c) \ominus(e, f, g)=(a-g, b-f, c-e)$

(iii) If $\widetilde{A}_{1}$ and $\widetilde{A}_{2}$ be two nonnegative triangular fuzzy numbers then

$$
\widetilde{A}_{1} \otimes \tilde{A}_{2}=\widetilde{A}_{2} \otimes \tilde{A}_{1}=(a e, b f, c g) .
$$


Remark 10. Let $\tilde{A}=(a, b, c)$ and $\tilde{X}=(x, y, z)$ be two unrestricted triangular fuzzy numbers. Then

$$
(a, b, c) \otimes(x, y, z)=\left\{\begin{array}{l}
\min (a x, c x), \text { by, } \max (a z, c z) \\
\quad \text { if }(a, b, c) \geq 0, \text { that is, if } a \geq 0, \\
\min (a z, c z), \text { by, } \max (a x, c x) \\
\quad \text { if }(a, b, c) \leq 0, \text { that is, if } c \leq 0, \\
\min (a z, c x), \text { by, } \max (c z, a x) \\
\text { otherwise, that is, if } a \leq 0 \leq c .
\end{array}\right.
$$

Remark 11. In this paper, minimum and maximum are represented by "min" and " $m a x "$, respectively, where

$$
\begin{aligned}
& \min (x, y)=\left(\frac{x+y}{2}\right)-\left|\frac{x-y}{2}\right|, \\
& \max (x, y)=\left(\frac{x+y}{2}\right)+\left|\frac{x-y}{2}\right| .
\end{aligned}
$$

\section{Limitations of the Existing Methods}

In this section, the limitations of the existing methods, for example, [2, 5-14, 17-29] are pointed out.

(1) The existing methods can be applied only for finding the solution of an $n \times n$ FFLS in which all the elements are nonnegative fuzzy numbers; that is, the existing methods presume the nonnegativity of the coefficient matrix and the solution vector. This restriction creates difficulty in using the existing methods to solve an FFLS occurring in real life situations as the nature of the unknown quantity cannot be known a priori and there exist several instances in real scenarios where the coefficients may not be entirely positive or nonnegative. The primary aim of this paper is to eliminate this restriction and to propose a concept and a computational paradigm to solve a fuzzy-based system of simultaneous equations without any nonnegative restrictions.

(2) There exist several methods like [5-11] that restrict the coefficient matrix to be crisp matrix and such systems are not fully fuzzy. Such systems will be entirely spanned by fully fuzzy linear systems. Some of the methods include $\alpha$-cuts to solve fuzzy linear equations which can be computationally simplified by using triangular fuzzy numbers as it is easier to operate with constants than variables.

(3) Using the existing methods it is not possible to check whether the obtained solution is unique or not. There may be several cases where the FFLS may generate trivial, unique or infinitely many solutions.

To overcome the above shortcomings, in Section 4, a new computational method is proposed for solving an FFLS with triangular fuzzy numbers.

\section{Proposed Method}

In this section, a new method is proposed to solve a fully fuzzy linear system with no restrictions on the parameters.

The steps of the proposed method are as follows.

Step 1. Substituting $\tilde{A}=\left(\tilde{a}_{i j}\right)_{m \times n}, \tilde{x}=\left(\tilde{x}_{j}\right)_{n \times 1}$, and $\widetilde{B}=$ $\left(\widetilde{B}_{i}\right)_{m \times 1}$ the $m \times n$ FFLS $\tilde{A} \otimes \tilde{x}=\widetilde{B}$, may be written as

$$
\sum_{j=1, \ldots, n}^{\oplus} \tilde{a}_{i j} \otimes \tilde{x}_{j}=\widetilde{B}_{i} \quad \forall i=1,2, \ldots, m .
$$

Step 2. If all the parameters $\tilde{a}_{i j}, \tilde{x}_{j}$, and $\tilde{b}_{i}$ are represented by triangular fuzzy numbers $\left(a_{i j}, b_{i j}, c_{i j}\right),\left(x_{j}, y_{j}, z_{j}\right)$, and $\left(B_{i}, G_{i}, H_{i}\right)$, respectively, then the FFLS, obtained in Step 1, may be written as

$$
\begin{array}{r}
\sum_{j=1, \ldots, n}^{\oplus}\left(a_{i j}, b_{i j}, c_{i j}\right) \otimes\left(x_{j}, y_{j}, z_{j}\right)=\left(B_{i}, G_{i}, H_{i}\right), \\
\forall i=1,2, \ldots, m .
\end{array}
$$

Step 3. Assuming $\left(a_{i j}, b_{i j}, c_{i j}\right) \otimes\left(x_{j}, y_{j}, z_{j}\right)=\left(f_{i j}, p_{i j}, q_{i j}\right)$, the FFLS, obtained in Step 2, may be written as

$$
\sum_{j=1, \ldots, n}^{\oplus}\left(f_{i j}, p_{i j}, q_{i j}\right)=\left(B_{i}, G_{i}, H_{i}\right) \quad \forall i=1,2, \ldots, m,
$$

where

$$
\left(f_{i j}, p_{i j}, q_{i j}\right)=\left(a_{i j}, b_{i j}, c_{i j}\right) \otimes\left(x_{j}, y_{j}, z_{j}\right) .
$$

As $\tilde{a}_{i j}$ and $\tilde{x}_{j}$ are both arbitrary fuzzy numbers, the above product can be defined in three subcases.

Case 1. If $\left(a_{i j}, b_{i j}, c_{i j}\right) \geq 0$, that is, $a_{i j} \geq 0$,

$$
\begin{aligned}
\left(f_{i j}, p_{i j}, q_{i j}\right)= & \left(\left(\frac{a_{i j}+c_{i j}}{2}\right) x_{j}-\left(\frac{c_{i j}-a_{i j}}{2}\right)\left|x_{j}\right|, b_{i j} y_{j},\right. \\
& \left.\left(\frac{a_{i j}+c_{i j}}{2}\right) z_{j}+\left(\frac{c_{i j}-a_{i j}}{2}\right)\left|z_{j}\right|\right) .
\end{aligned}
$$

Using Definition 7, the above equation may be written as,

$$
\begin{gathered}
f_{i j}=\left(\frac{a_{i j}+c_{i j}}{2}\right) x_{j}-\left(\frac{c_{i j}-a_{i j}}{2}\right)\left|x_{j}\right|, \\
p_{i j}=b_{i j} y_{j}, \\
q_{i j}=\left(\frac{a_{i j}+c_{i j}}{2}\right) z_{j}+\left(\frac{c_{i j}-a_{i j}}{2}\right)\left|z_{j}\right| .
\end{gathered}
$$

To solve this system we define variables $x_{j}^{\prime}$ and $x_{j}^{\prime \prime}$ as

$$
\begin{gathered}
x_{j}^{\prime}= \begin{cases}x_{j} & \text { if } x_{j}>0, \\
0 & \text { otherwise }\end{cases} \\
x_{j}^{\prime \prime}= \begin{cases}-x_{j} & \text { if } x_{j}<0, \\
0 & \text { otherwise }\end{cases}
\end{gathered}
$$


Hence substitute $x_{j}=x_{j}^{\prime}-x_{j}^{\prime \prime}$ and $\left|x_{j}\right|=x_{j}^{\prime}+x_{j}^{\prime \prime}$; similarly define variables $z_{j}^{\prime}$ and $z_{j}^{\prime \prime}$; substitute $z_{j}=z_{j}^{\prime}-z_{j}^{\prime \prime}$ and $\left|z_{j}\right|=$ $z_{j}^{\prime}+z_{j}^{\prime \prime}$ where $x_{j}^{\prime}, x_{j}^{\prime \prime}, z_{j}^{\prime}, z_{j}^{\prime \prime} \geq 0$. Therefore we obtain the following linear system of equation:

$$
\begin{gathered}
f_{i j}=\left(\frac{a_{i j}+c_{i j}}{2}\right)\left(x_{j}^{\prime}-x_{j}^{\prime \prime}\right)-\left(\frac{c_{i j}-a_{i j}}{2}\right)\left(x_{j}^{\prime}+x_{j}^{\prime \prime}\right), \\
p_{i j}=b_{i j} y_{j}, \\
q_{i j}=\left(\frac{a_{i j}+c_{i j}}{2}\right)\left(z_{j}^{\prime}-z_{j}^{\prime \prime}\right)+\left(\frac{c_{i j}-a_{i j}}{2}\right)\left(z_{j}^{\prime}+z_{j}^{\prime \prime}\right) .
\end{gathered}
$$

Case 2. If $\left(a_{i j}, b_{i j}, c_{i j}\right) \leq 0$, that is, $c_{i j} \leq 0$,

$$
\begin{aligned}
\left(f_{i j}, p_{i j}, q_{i j}\right)= & \left(\left(\frac{a_{i j}+c_{i j}}{2}\right) z_{j}-\left(\frac{c_{i j}-a_{i j}}{2}\right)\left|z_{j}\right|, b_{i j} y_{j},\right. \\
& \left.\left(\frac{a_{i j}+c_{i j}}{2}\right) x_{j}+\left(\frac{c_{i j}-a_{i j}}{2}\right)\left|x_{j}\right|\right) .
\end{aligned}
$$

This can be similarly reduced as follows.

Putting $x_{j}=x_{j}^{\prime}-x_{j}^{\prime \prime}$ and $\left|x_{j}\right|=x_{j}^{\prime}+x_{j}^{\prime \prime}, z_{j}=z_{j}^{\prime}-z_{j}^{\prime \prime}$, and $\left|z_{j}\right|=z_{j}^{\prime}+z_{j}^{\prime \prime}$, where $x_{j}^{\prime}, x_{j}^{\prime \prime}, z_{j}^{\prime}, z_{j}^{\prime \prime} \geq 0$. Thus

$$
\begin{gathered}
f_{i j}=\left(\frac{a_{i j}+c_{i j}}{2}\right)\left(z_{j}^{\prime}-z_{j}^{\prime \prime}\right)-\left(\frac{c_{i j}-a_{i j}}{2}\right)\left(z_{j}^{\prime}+z_{j}^{\prime \prime}\right), \\
p_{i j}=b_{i j} y_{j}, \\
q_{i j}=\left(\frac{a_{i j}+c_{i j}}{2}\right)\left(x_{j}^{\prime}-x_{j}^{\prime \prime}\right)+\left(\frac{c_{i j}-a_{i j}}{2}\right)\left(x_{j}^{\prime}+x_{j}^{\prime \prime}\right) .
\end{gathered}
$$

Case 3. If $\left(a_{i j}, b_{i j}, c_{i j}\right) \approx 0$, that is, $a_{i j}<0<c_{i j}$,

$$
\begin{aligned}
\left(f_{i j}, p_{i j}, q_{i j}\right)= & \left(\left(\frac{a_{i j} z_{j}+c_{i j} x_{j}}{2}\right)-\left|\frac{c_{i j} x_{j}-a_{i j} z_{j}}{2}\right|, b_{i j} y_{j},\right. \\
& \left.\left(\frac{a_{i j} x_{j}+c_{i j} z_{j}}{2}\right)+\left|\frac{c_{i j} z_{j}-a_{i j} x_{j}}{2}\right|\right) .
\end{aligned}
$$

Using Definition 5, the above equation may be written as

$$
\begin{aligned}
& f_{i j}=\left(\frac{a_{i j} z_{j}+c_{i j} x_{j}}{2}\right)-\left|\frac{c_{i j} x_{j}-a_{i j} z_{j}}{2}\right|, \\
& p_{i j}=b_{i j} y_{j}, \\
& q_{i j}=\left(\frac{a_{i j} x_{j}+c_{i j} z_{j}}{2}\right)+\left|\frac{c_{i j} z_{j}-a_{i j} x_{j}}{2}\right| .
\end{aligned}
$$

This can be further reduced as follows.

Putting $x_{j}=x_{j}^{\prime}-x_{j}^{\prime \prime}$ and $\left|x_{j}\right|=x_{j}^{\prime}+x_{j}^{\prime \prime}, z_{j}=z_{j}^{\prime}-z_{j}^{\prime \prime}$ and $\left|z_{j}\right|=z_{j}^{\prime}+z_{j}^{\prime \prime}, c_{i j} x_{j}-a_{i j} z_{j}=u_{i j}^{\prime}-u_{i j}^{\prime \prime}$ and $\left|c_{i j} x_{j}-a_{i j} z_{j}\right|=$ $u_{i j}^{\prime}+u_{i j}^{\prime \prime}, c_{i j} z_{j}-a_{i j} x_{j}=v_{i j}^{\prime}-v_{i j}^{\prime \prime}$ and $\left|c_{i j} z_{j}-a_{i j} x_{j}\right|=v_{i j}^{\prime}+v_{i j}^{\prime \prime}$, where $x_{j}^{\prime}, x_{j}^{\prime \prime}, z_{j}^{\prime}, z_{j}^{\prime \prime}, u_{i j}^{\prime}, u_{i j}^{\prime \prime}, v_{i j}^{\prime}, v_{i j}^{\prime \prime} \geq 0$.
On simplifying we obtain

$$
\begin{gathered}
f_{i j}=\left(\frac{a_{i j}\left(z_{j}^{\prime}-z_{j}^{\prime \prime}\right)+c_{i j}\left(x_{j}^{\prime}-x_{j}^{\prime \prime}\right)}{2}\right)-\left(\frac{u_{i j}^{\prime}+u_{i j}^{\prime \prime}}{2}\right), \\
p_{i j}=b_{i j} y_{j}, \\
q_{i j}=\left(\frac{a_{i j}\left(x_{j}^{\prime}-x_{j}^{\prime \prime}\right)+c_{i j}\left(z_{j}^{\prime}-z_{j}^{\prime \prime}\right)}{2}\right)+\left(\frac{v_{i j}^{\prime}+v_{i j}^{\prime \prime}}{2}\right) .
\end{gathered}
$$

Step 4. Having broken down the FFLS from Step 3 into a set of linear equations, rewrite the given system as:

$$
\left(\sum_{j=1}^{n} f_{i j}, \sum_{j=1}^{n} p_{i j}, \sum_{j=1}^{n} q_{i j}\right)=\left(B_{i}, G_{i}, H_{i}\right) \quad \forall i=1,2, \ldots, m .
$$

Or equivalently

$$
\begin{aligned}
& \sum_{j=1}^{n} f_{i j}=B_{i} ; \quad i=1,2, \ldots, m, \\
& \sum_{j=1}^{n} p_{i j}=G_{i} ; \quad i=1,2, \ldots, m, \\
& \sum_{j=i}^{n} q_{i j}=H_{i} ; \quad i=1,2, \ldots, m,
\end{aligned}
$$

with appropriate constraints obtained from Step 3.

Step 5. In order to solve the system obtained in Step 4 we decompose the FFLS into an equivalent linear programming problem LPP. For notational convenience we construct the following step functions:

$$
\begin{aligned}
& \varepsilon_{1}(\tilde{A}=(a, b, c))= \begin{cases}1 & \text { if } \tilde{A} \geq 0, \text { that is, } a \geq 0, \\
0 & \text { otherwise, }\end{cases} \\
& \varepsilon_{2}(\tilde{A}=(a, b, c))= \begin{cases}1 & \text { if } \tilde{A} \leq 0, \text { that is, } c<0, \\
0 & \text { otherwise, }\end{cases}
\end{aligned}
$$

$$
\varepsilon_{3}(\tilde{A}=(a, b, c))= \begin{cases}1 & \text { if } \tilde{A} \approx 0, \text { that is, } a<0<c, \\ 0 & \text { otherwise. }\end{cases}
$$

The equivalent LPP can thus be directly written as: Minimize

$$
\bar{R}=\sum_{i=1}^{m} R_{i}+\sum_{i=1}^{m} R_{i}^{\prime}+\sum_{i=1}^{m} R_{i}^{\prime \prime}
$$

subject to the following constraints.

First Set of Constraints:

$$
\sum_{j=1}^{n} b_{i j} y_{j}+R_{i}=G_{i}, \quad i=1,2, \ldots, m .
$$


Second Set of Constraints:

$$
\begin{array}{r}
\sum_{j=1}^{n} \varepsilon_{1}\left(\tilde{a}_{i j}\right)\left(a_{i j} x_{j}^{\prime}-c_{i j} x_{j}^{\prime \prime}\right)+\sum_{j=1}^{n} \varepsilon_{2}\left(\tilde{a}_{i j}\right)\left(a_{i j} z_{j}^{\prime}-c_{i j} z_{j}^{\prime \prime}\right) \\
+\sum_{j=1}^{n} \varepsilon_{3}\left(\tilde{a}_{i j}\right)\left(0.5\left(a_{i j}\left(z_{j}^{\prime}-z_{j}^{\prime \prime}\right)+c_{i j}\left(x_{j}^{\prime}-x_{j}^{\prime \prime}\right)\right)\right. \\
\left.-0.5\left(u_{i j}^{\prime}+u_{i j}^{\prime \prime}\right)\right)+R_{i}^{\prime}=B_{i} \\
\forall i=1,2, \ldots, m .
\end{array}
$$

Third Set of Constraints:

$$
\begin{gathered}
\sum_{j=1}^{n} \varepsilon_{1}\left(\tilde{a}_{i j}\right)\left(c_{i j} z_{j}^{\prime}-a_{i j} z_{j}^{\prime \prime}\right)+\sum_{j=1}^{n} \varepsilon_{2}\left(\tilde{a}_{i j}\right)\left(c_{i j} x_{j}^{\prime}-a_{i j} x_{j}^{\prime \prime}\right) \\
+\sum_{j=1}^{n} \varepsilon_{3}\left(\tilde{a}_{i j}\right)\left(0.5\left(c_{i j}\left(z_{j}^{\prime}-z_{j}^{\prime \prime}\right)+a_{i j}\left(x_{j}^{\prime}-x_{j}^{\prime \prime}\right)\right)\right. \\
\left.+0.5\left(v_{i j}^{\prime}+v_{i j}^{\prime \prime}\right)\right)+R_{i}^{\prime \prime}=H_{i} . \\
\forall i=1,2, \ldots, m .
\end{gathered}
$$

Fourth Set of Constraints:

$$
\begin{gathered}
\varepsilon_{3}\left(\tilde{a}_{i j}\right)\left(c_{i j} x_{j}-a_{i j} z_{j}-u_{i j}^{\prime}+u_{i j}^{\prime \prime}\right)=0, \\
i=1,2, \ldots m, j=1,2, \ldots, n .
\end{gathered}
$$

Fifth Set of Constraint:

$$
\begin{gathered}
\varepsilon_{3}\left(\tilde{a}_{i j}\right)\left(c_{i j} z_{j}-a_{i j} x_{j}-v_{i j}^{\prime}+v_{i j}^{\prime \prime}\right)=0, \\
i=1,2, \ldots m, j=1,2, \ldots, n .
\end{gathered}
$$

Nonnegative Constraint:

$$
\begin{aligned}
& x_{j}^{\prime}, x_{j}^{\prime \prime}, \varepsilon_{3}\left(\tilde{a}_{i j}\right) u_{i j}^{\prime}, \varepsilon_{3}\left(\tilde{a}_{i j}\right) u_{i j}^{\prime \prime}, \varepsilon_{3}\left(\tilde{a}_{i j}\right) v_{i j}^{\prime}, \\
& \varepsilon_{3}\left(\tilde{a}_{i j}\right) v_{i j}^{\prime \prime}, z_{j}^{\prime}, z_{j}^{\prime \prime}, R_{i}, R_{i}^{\prime}, R_{i}^{\prime \prime} \geq 0, \\
& i=1,2, \ldots m, j=1,2, \ldots, n .
\end{aligned}
$$

Step 6. Solve the above LPP and put the above values in

$$
\tilde{x}_{j}=\left(x_{j}^{\prime}-x_{j}^{\prime \prime}, y_{j}, z_{j}^{\prime}-z_{j}^{\prime \prime}\right) \quad \forall j=1,2, \ldots, n,
$$

to find the solution of FFLS. The solution would be termed as (feasible) strong fuzzy solution if $y_{j}-x_{j}^{\prime}+x_{j}^{\prime \prime} \geq 0$ and $z_{j}^{\prime}-z_{j}^{\prime \prime}-y_{j} \geq 0$. Otherwise the solution would be termed as (infeasible) weak fuzzy solution.

Remark 12. The necessary condition for the above LPP to generate a feasible solution is that the value of the objective function $R$ must be zero, that is, $\sum_{i=1}^{m} R_{i}+\sum_{i=1}^{m} R_{i}^{\prime}+\sum_{i=1}^{m} R_{i}^{\prime \prime}=$ 0.
Remark 13. The sufficient conditions for the solution to be termed as a strong (feasible) solution for $i=1,2, \ldots m, j=$ $1,2, \ldots, n$, are as follows:

$$
\begin{aligned}
& \text { (1) } y_{j}-x_{j}^{\prime}+x_{j}^{\prime \prime} \geq 0 \text { and } z_{j}^{\prime}-z_{j}^{\prime \prime}-y_{j} \geq 0, \\
& \text { (2) } x_{j}^{\prime} \cdot x_{j}^{\prime \prime}=u_{i j}^{\prime} \cdot u_{i j}^{\prime \prime}=v_{i j}^{\prime} \cdot v_{i j}^{\prime \prime}=z_{j}^{\prime} \cdot z_{j}^{\prime \prime}=R=0 .
\end{aligned}
$$

Remark 14. The nature of the solutions of the FFLS depends upon the nature of the solutions of the LPP which may be unique, trivial, or infinitely many, that is, the FFLS may yield no solution, unique solution, or infinitely many solutions.

Remark 15. The decomposition of the $m \times n$ FFLS results into an LPP with a minimum number of $3 m$ constraints (when all the coefficients are either nonnegative or nonpositive) and a maximum number of $3 m+2 m n$ constraints (when all the coefficients are neither negative nor positive).

\section{Numerical Example}

In this section, to illustrate the proposed method and also to show the advantages of the proposed method we solve three FFLSs. We take two FFLSs which cannot be solved by any of the available methods in the literature due to the restrictions imposed by the methods. Also, we solve one FFLSs randomly chosen from literature and apply our method to show that the proposed method can equivalently solve the examples from literature. Hence the FFLS that we solve belong to the following categories:

(1) a square FFLS with unrestricted fuzzy variables,

(2) a nonsquare FFLS with unrestricted fuzzy variables that generates infinite solutions,

(3) a square FFLS with restriction of crispness of the coefficient matrix randomly chosen from the literature [5].

Example 1. Solve the following FFLS by the proposed method:

$$
\left[\begin{array}{cc}
(-2,3,4) & (-2,2,3) \\
(1,2,2) & (4,4,5)
\end{array}\right]\left[\begin{array}{l}
\tilde{x}_{1} \\
\tilde{x}_{2}
\end{array}\right]=\left[\begin{array}{l}
(-13,8,14) \\
(-14,8,14)
\end{array}\right],
$$

where $\tilde{x}_{1}$ and $\tilde{x}_{2}$ are arbitrary triangular fuzzy numbers.

\section{Solution 1.}

Step 1. The chosen FFLS is as follows:

$$
\begin{gathered}
(-2,3,4) \otimes \tilde{x}_{1} \oplus(-2,2,3) \otimes \tilde{x}_{2}=(-13,8,14), \\
(1,2,2) \otimes \tilde{x}_{1} \oplus(4,4,5) \otimes \tilde{x}_{2}=(-14,8,14),
\end{gathered}
$$

where $\tilde{x}_{1}, \tilde{x}_{2}$ are unrestricted triangular fuzzy numbers. 
Step 2. Assuming $\tilde{x}_{1}=\left(x_{1}, y_{1}, z_{1}\right)$ and $\tilde{x}_{2}=\left(x_{2}, y_{2}, z_{2}\right)$ the FFLS, chosen in Step 1, may be written as

$$
\begin{aligned}
(-2,3,4) & \otimes\left(x_{1}, y_{1}, z_{1}\right) \oplus(-2,2,3) \otimes\left(x_{2}, y_{2}, z_{2}\right) \\
= & (-13,8,14) \\
(1,2,2) & \otimes\left(x_{1}, y_{1}, z_{1}\right) \oplus(4,4,5) \otimes\left(x_{2}, y_{2}, z_{2}\right) \\
= & (-14,8,14),
\end{aligned}
$$

where $\left(x_{1}, y_{1}, z_{1}\right)$ and $\left(x_{2}, y_{2}, z_{2}\right)$ are unrestricted triangular fuzzy numbers.

Step 3. Using Step 3 we may obtain $\left(f_{i j}, p_{i j}, q_{i j}\right)$ as follows:

$$
\begin{gathered}
\left(f_{11}, p_{11}, q_{11}\right)=(-2,3,4) \otimes\left(x_{1}, y_{1}, z_{1}\right), \\
f_{11}=\left(\frac{a_{11}\left(z_{1}^{\prime}-z_{1}^{\prime \prime}\right)+c_{11}\left(x_{1}^{\prime}-x_{1}^{\prime \prime}\right)}{2}\right)-\left(\frac{u_{11}^{\prime}+u_{11}^{\prime \prime}}{2}\right), \\
p_{11}=b_{11} y_{1}, \\
q_{11}=\left(\frac{a_{11}\left(x_{1}^{\prime}-x_{1}^{\prime \prime}\right)+c_{11}\left(z_{1}^{\prime}-z_{1}^{\prime \prime}\right)}{2}\right)+\left(\frac{v_{11}^{\prime}+v_{11}^{\prime \prime}}{2}\right) .
\end{gathered}
$$

Or we may have

$$
\begin{gathered}
p_{11}=3 y_{1}, \\
f_{11}=\frac{-2\left(z_{1}^{\prime}-z_{1}^{\prime \prime}\right)+4\left(x_{1}^{\prime}-x_{1}^{\prime \prime}\right)}{2}-\left(\frac{u_{11}^{\prime}+u_{11}^{\prime \prime}}{2}\right), \\
q_{11}=\frac{4\left(z_{1}^{\prime}-z_{1}^{\prime \prime}\right)-2\left(x_{1}^{\prime}-x_{1}^{\prime \prime}\right)}{2}+\left(\frac{v_{11}^{\prime}+v_{11}^{\prime \prime}}{2}\right),
\end{gathered}
$$

with two additional constraints:

$$
\begin{aligned}
& 2\left(z_{1}^{\prime}-z_{1}^{\prime \prime}\right)+4\left(x_{1}^{\prime}-x_{1}^{\prime \prime}\right)-u_{11}^{\prime}+u_{11}^{\prime \prime}=0, \\
& 2\left(x_{1}^{\prime}-x_{1}^{\prime \prime}\right)+4\left(z_{1}^{\prime}-z_{1}^{\prime \prime}\right)-v_{11}^{\prime}+v_{11}^{\prime \prime}=0 .
\end{aligned}
$$

Similarly we obtain the other coefficients as

$$
\begin{gathered}
p_{12}=2 y_{2}, \\
f_{12}=\frac{-2\left(z_{2}^{\prime}-z_{2}^{\prime \prime}\right)+3\left(x_{2}^{\prime}-x_{2}^{\prime \prime}\right)}{2}-\left(\frac{u_{12}^{\prime}+u_{12}^{\prime \prime}}{2}\right), \\
q_{12}=\frac{3\left(z_{2}^{\prime}-z_{2}^{\prime \prime}\right)-2\left(x_{2}^{\prime}-x_{2}^{\prime \prime}\right)}{2}+\left(\frac{v_{12}^{\prime}+v_{12}^{\prime \prime}}{2}\right),
\end{gathered}
$$

with two additional equations:

$$
\begin{gathered}
2\left(z_{2}^{\prime}-z_{2}^{\prime \prime}\right)+3\left(x_{2}^{\prime}-x_{2}^{\prime \prime}\right)-u_{12}^{\prime}+u_{12}^{\prime \prime}=0 \\
2\left(x_{2}^{\prime}-x_{2}^{\prime \prime}\right)+3\left(z_{2}^{\prime}-z_{2}^{\prime \prime}\right)-v_{12}^{\prime}+v_{12}^{\prime \prime}=0 \\
p_{21}=2 x_{1} \\
f_{21}=x_{1}^{\prime}-2 x_{1}^{\prime \prime} \\
q_{21}=2 z_{1}^{\prime}-z_{1}^{\prime \prime} \\
p_{22}=4 x_{2} \\
f_{22}=4 x_{2}^{\prime}-5 x_{2}^{\prime \prime} \\
q_{22}=5 z_{2}^{\prime}-4 z_{2}^{\prime \prime} .
\end{gathered}
$$

Step 4. Using Step 4 of the proposed method the FFLS obtained in Step 3 may be written as

$$
\begin{gathered}
3 y_{1}+2 y_{2}=8, \\
2 y_{1}+4 y_{2}=8, \\
f_{11}+f_{12}=-13, \\
f_{21}+f_{22}=-14, \\
q_{11}+q_{12}=14, \\
q_{21}+q_{22}=14 .
\end{gathered}
$$

Step 5. Convert the system of equation as a linear programming problem as follows.

Minimize $R=R_{1}+R_{2}+R_{1}^{\prime}+R_{2}^{\prime}+R_{1}^{\prime \prime}+R_{2}^{\prime \prime}$ subject to the following 10 constraints:

$$
\begin{gathered}
3 y_{1}+2 y_{2}+R_{1}=8, \\
2 y_{1}+4 y_{2}+R_{2}=8 \\
-z_{1}^{\prime}+z_{1}^{\prime \prime}+2 x_{1}^{\prime}-2 x_{1}^{\prime \prime}-0.5 u_{11}^{\prime}-0.5 u_{11}^{\prime \prime}-z_{2}^{\prime}+z_{2}^{\prime \prime}+1.5 x_{2}^{\prime} \\
-1.5 x_{2}^{\prime \prime}-0.5 u_{12}^{\prime}-0.5 u_{12}^{\prime \prime}+R_{1}^{\prime}=-13, \\
x_{1}^{\prime}-2 x_{1}^{\prime \prime}+4 x_{2}^{\prime}-5 x_{2}^{\prime \prime}+R_{2}^{\prime}=-14, \\
2 z_{1}^{\prime \prime}-x_{1}^{\prime}+x_{1}^{\prime \prime}+0.5 v_{11}^{\prime}+0.5 v_{11}^{\prime \prime}+1.5 z_{2}^{\prime}-1.5 z_{2}^{\prime \prime} \\
-x_{2}^{\prime}+x_{2}^{\prime \prime}+0.5 v_{12}^{\prime}+0.5 v_{12}^{\prime \prime}+R_{1}^{\prime \prime}=14, \\
2 z_{1}^{\prime}-z_{1}^{\prime \prime}+5 z_{2}^{\prime}-4 z_{2}^{\prime \prime}+R_{2}^{\prime \prime}=14, \\
2\left(z_{1}^{\prime}-z_{1}^{\prime \prime}\right)+4\left(x_{1}^{\prime}-x_{1}^{\prime \prime}\right)-u_{11}^{\prime}+u_{11}^{\prime \prime}=0 \\
2\left(x_{1}^{\prime}-x_{1}^{\prime \prime}\right)+4\left(z_{1}^{\prime}-z_{1}^{\prime \prime}\right)-v_{11}^{\prime}+v_{11}^{\prime \prime}=0 \\
2\left(z_{2}^{\prime}-z_{2}^{\prime \prime}\right)+3\left(x_{2}^{\prime}-x_{2}^{\prime \prime}\right)-u_{12}^{\prime}+u_{12}^{\prime \prime}=0 \\
2\left(x_{2}^{\prime}-x_{2}^{\prime \prime}\right)+3\left(z_{2}^{\prime}-z_{2}^{\prime \prime}\right)-v_{12}^{\prime}+v_{12}^{\prime \prime}=0, \\
x_{1}^{\prime}, x_{1}^{\prime \prime}, x_{2}^{\prime}, x_{2}^{\prime \prime}, u_{11}^{\prime}, u_{11}^{\prime \prime}, v_{11}^{\prime}, v_{11}^{\prime \prime}, z_{1}^{\prime}, z_{1}^{\prime \prime}, z_{2}^{\prime}, z_{2}^{\prime \prime}, \\
u_{12}^{\prime}, u_{12}^{\prime \prime}, v_{12}^{\prime}, v_{12}^{\prime \prime}, R_{1}, R_{2}, R_{1}^{\prime}, R_{2}^{\prime}, R_{1}^{\prime \prime}, R_{2}^{\prime \prime} \geq 0 .
\end{gathered}
$$

On solving the above LPP, we get $y_{1}=2, y_{2}=1, x_{1}^{\prime}=1$, $x_{1}^{\prime \prime}=0, u_{11}^{\prime}=8, u_{11}^{\prime \prime}=0, v_{11}^{\prime}=10, v_{11}^{\prime \prime}=0, x_{2}^{\prime}=0, x_{2}^{\prime \prime}=3$, $z_{1}^{\prime}=2, z_{1}^{\prime \prime}=0, z_{2}^{\prime}=2, z_{2}^{\prime \prime}=0, u_{12}^{\prime}=0, u_{12}^{\prime \prime}=5, v_{12}^{\prime}=0$, $v_{12}^{\prime \prime}=0, R_{1}=0, R_{2}=0, R_{1}^{\prime}=0, R_{2}^{\prime}=0, R_{1}^{\prime \prime}=0$, and $R_{2}^{\prime \prime}=0$.

Step 6. Put the values of $x_{j}^{\prime}, x_{j}^{\prime \prime}, z_{j}^{\prime}, z_{j}^{\prime \prime}, y_{j}$, obtained from Step 5 , in $\tilde{x}_{j}=\left(x_{j}^{\prime}-x_{j}^{\prime \prime}, y_{j}, z_{j}^{\prime}-z_{j}^{\prime \prime}\right)$ for all $j=1,2, \ldots, n$. We find the solution of FFLS as

$$
\tilde{x}_{1}=(1,2,2), \quad \tilde{x}_{2}=(-3,1,2) .
$$

Clearly, the FFLS has a feasible and a strong fuzzy solution. 
Example 2. Solve the following $2 \times 3$ FFLS:

$$
\begin{gathered}
{\left[\begin{array}{ccc}
(1,2,3) & (-2,-1,-1) & (2,3,4) \\
(-3,-2,-1) & (2,4,5) & (3,4,5)
\end{array}\right]\left[\begin{array}{c}
\tilde{x}_{1} \\
\tilde{x}_{2} \\
\tilde{x}_{3}
\end{array}\right]} \\
=\left[\begin{array}{c}
(-7,0,8) \\
(-26,-10,-4)
\end{array}\right] .
\end{gathered}
$$

Solution 2. Clearly this is a nonsquare FFLS with arbitrary triangular fuzzy numbers as coefficients and hence cannot be solved by any of the existing methods. We apply our method to solve this system.

Rewrite the FFLS as

$$
\begin{aligned}
& (1,2,3) \otimes\left(x_{1}, y_{1}, z_{1}\right) \oplus(-2,-1,-1) \otimes\left(x_{2}, y_{2}, z_{2}\right) \\
& \oplus(2,3,4) \otimes\left(x_{3}, y_{3}, z_{3}\right)=(-7,0,8), \\
& (-3,-2,-1) \otimes\left(x_{1}, y_{1}, z_{1}\right) \oplus(2,4,5) \otimes\left(x_{2}, y_{2}, z_{2}\right) \\
& \oplus(3,4,5) \otimes\left(x_{3}, y_{3}, z_{3}\right)=(-26,-10,-4) .
\end{aligned}
$$

Using the proposed method we obtain the following LPP:

Minimize $R=R_{1}+R_{2}+R_{1}^{\prime}+R_{2}^{\prime}+R_{1}^{\prime \prime}+R_{2}^{\prime \prime}$ subject to the following 6 constraints:

$$
\begin{gathered}
2 y_{1}-y_{2}+3 y_{3}+R_{1}=0, \\
-2 y_{1}+4 y_{2}+4 y_{3}+R_{2}=-10, \\
x_{1}^{\prime}-3 x_{1}^{\prime \prime}-2 z_{2}^{\prime}+z_{2}^{\prime \prime}+2 x_{3}^{\prime}-4 x_{3}^{\prime \prime}+R_{1}^{\prime}=-7, \\
3 z_{1}^{\prime}-z_{1}^{\prime \prime}-x_{2}^{\prime}+2 x_{2}^{\prime \prime}+4 z_{3}^{\prime}-2 z_{3}^{\prime \prime}+R_{1}^{\prime \prime}=8, \\
-3 z_{1}^{\prime}+z_{1}^{\prime \prime}+2 x_{2}^{\prime}-5 x_{2}^{\prime \prime}+3 x_{3}^{\prime}-5 x_{3}^{\prime \prime}+R_{2}^{\prime}=-26, \\
-x_{1}^{\prime}+3 x_{1}^{\prime \prime}+5 z_{2}^{\prime}-2 z_{2}^{\prime \prime}+5 z_{3}^{\prime}-3 z_{3}^{\prime \prime}+R_{2}^{\prime \prime}=-4, \\
x_{1}^{\prime}, x_{1}^{\prime \prime}, x_{2}^{\prime}, x_{2}^{\prime \prime}, x_{3}^{\prime}, x_{3}^{\prime \prime}, y_{1}^{\prime}, y_{1}^{\prime \prime}, y_{2}^{\prime}, y_{2}^{\prime \prime}, \\
y_{3}^{\prime}, y_{3}^{\prime \prime}, R_{1}, R_{2}, R_{1}^{\prime}, R_{2}^{\prime}, R_{1}^{\prime \prime}, R_{2}^{\prime \prime} \geq 0 .
\end{gathered}
$$

The above LPP on solving by traditional algebra yields many solutions and one of the feasible solutions is $y_{1}=1, y_{2}=-1$, $y_{3}=-1, x_{1}^{\prime}=1, x_{1}^{\prime \prime}=0, x_{2}^{\prime}=0, x_{2}^{\prime \prime}=2, x_{3}^{\prime}=0, x_{3}^{\prime \prime}=2$, $z_{1}^{\prime}=2, z_{1}^{\prime \prime}=0, z_{2}^{\prime}=0, z_{2}^{\prime \prime}=0, z_{3}^{\prime}=0, z_{3}^{\prime \prime}=1, R_{1}=0$, $R_{2}=0, R_{1}^{\prime}=0, R_{2}^{\prime}=0, R_{1}^{\prime \prime}=0$, and $R_{2}^{\prime \prime}=0$.

Put the values of $x_{j}^{\prime}, x_{j}^{\prime \prime}, z_{j}^{\prime}, z_{j}^{\prime \prime}, y_{j}$ obtained from Step 5, in $\tilde{x}_{j}=\left(x_{j}^{\prime}-x_{j}^{\prime \prime}, y_{j}, z_{j}^{\prime}-z_{j}^{\prime \prime}\right)$ for all $j=1,2, \ldots, n$. we find the solution of FFLS as

$$
\tilde{x}_{1}=(1,1,2), \quad \tilde{x}_{2}=(-2,-1,0), \quad \tilde{x}_{3}=(-2,-1,-1) .
$$

Clearly, the obtained solution is a feasible (strong) fuzzy solution.

Example 3. Solve the following $2 \times 2$ fuzzy system randomly taken from [5]:

$$
\begin{gathered}
x_{1}-x_{2}=(r, 2-r), \\
x_{1}+3 x_{2}=(4+r, 7-2 r) .
\end{gathered}
$$

Solution 3. The fuzzy linear system is based upon the concept of $\alpha$-cuts and has assumed restricted nature of FFLS by considering the coefficients crisp rather than fuzzy. We first convert this system into an FFLS with triangular fuzzy numbers and solve it by the proposed method to obtain the same result. The above system can be rewritten as

$$
\left[\begin{array}{cc}
(1,1,1) & (-1,-1,-1) \\
(1,1,1) & (3,3,3)
\end{array}\right]\left[\begin{array}{l}
\tilde{x}_{1} \\
\tilde{x}_{2}
\end{array}\right]=\left[\begin{array}{l}
(0,1,2) \\
(4,5,7)
\end{array}\right],
$$

where $\tilde{x}_{1}$ and $\tilde{x}_{2}$ are arbitrary triangular fuzzy numbers.

Assuming $\tilde{x}_{1}=\left(x_{1}, y_{1}, z_{1}\right)$ and $\tilde{x}_{2}=\left(x_{2}, y_{2}, z_{2}\right)$, the FFLS may be written as

$$
\begin{aligned}
(1,1,1) & \otimes\left(x_{1}, y_{1}, z_{1}\right) \oplus(-1,-1,-1) \otimes\left(x_{2}, y_{2}, z_{2}\right) \\
= & (0,1,2) \\
(1,1,1) & \otimes\left(x_{1}, y_{1}, z_{1}\right) \oplus(3,3,3) \otimes\left(x_{2}, y_{2}, z_{2}\right) \\
= & (4,5,7),
\end{aligned}
$$

where $\left(x_{1}, y_{1}, z_{1}\right)$ and $\left(x_{2}, y_{2}, z_{2}\right)$ are unrestricted triangular fuzzy numbers.

Using the proposed method we obtain the following LPP. Minimize $R=R_{1}+R_{2}+R_{1}^{\prime}+R_{2}^{\prime}+R_{1}^{\prime \prime}+R_{2}^{\prime \prime}$ subject to the following 6 constraints:

$$
\begin{gathered}
y_{1}-y_{2}+R_{1}=1, \\
y_{1}+3 y_{2}+R_{2}=5, \\
x_{1}^{\prime}-x_{1}^{\prime \prime}-z_{2}^{\prime}+z_{2}^{\prime \prime}+R_{1}^{\prime}=0, \\
x_{1}^{\prime}-x_{1}^{\prime \prime}+3 x_{2}^{\prime}-3 x_{3}^{\prime \prime}+R_{2}^{\prime}=4, \\
z_{1}^{\prime}-z_{1}^{\prime \prime}-x_{2}^{\prime}+x_{2}^{\prime \prime}+R_{1}^{\prime \prime}=2, \\
z_{1}^{\prime}-z_{1}^{\prime \prime}+3 z_{2}^{\prime}-3 z_{2}^{\prime \prime}+R_{2}^{\prime \prime}=7, \\
x_{1}^{\prime}, x_{1}^{\prime \prime}, x_{2}^{\prime}, x_{2}^{\prime \prime}, y_{1}^{\prime}, y_{1}^{\prime \prime}, y_{2}^{\prime}, y_{2}^{\prime \prime}, R_{1}, R_{2}, R_{1}^{\prime}, R_{2}^{\prime}, R_{1}^{\prime \prime}, R_{2}^{\prime \prime} \geq 0 .
\end{gathered}
$$

The above LPP on solving by traditional algebra yields the following solution: $y_{1}=2, y_{2}=1, x_{1}^{\prime}=1.375, x_{1}^{\prime \prime}=0$, $x_{2}^{\prime}=0.875, x_{2}^{\prime \prime}=0, z_{1}^{\prime}=2.875, z_{1}^{\prime \prime}=0, z_{2}^{\prime}=1.375, z_{2}^{\prime \prime}=0$, $R_{1}=0, R_{2}=0, R_{1}^{\prime}=0, R_{2}^{\prime}=0, R_{1}^{\prime \prime}=0$, and $R_{2}^{\prime \prime}=0$.

Substituting the values of $x_{j}^{\prime}, x_{j}^{\prime \prime}, z_{j}^{\prime}, z_{j}^{\prime \prime}, y_{j}$, obtained from Step 5 , in $\tilde{x}_{j}=\left(x_{j}^{\prime}-x_{j}^{\prime \prime}, y_{j}, z_{j}^{\prime}-z_{j}^{\prime \prime}\right)$ for all $j=1,2, \ldots, n$ we find the solution of FFLS as

$$
\tilde{x}_{1}=(1.375,2,2.875), \quad \tilde{x}_{2}=(0.875,1,1.375) .
$$

Clearly, the obtained solution is exactly the same as in [5] and is clearly feasible.

\section{Conclusion}

In this paper, we propose a new method for solving FFLS with arbitrary coefficients that not only overcomes the limitations of the several existing methods available in the literature like $[2,5-14,17-29]$ but also amplifies the scope 
of incorporating fuzzy linear equation in real scenarios. The proposed method can successfully solve the following types of FFLS:

(i) FFLS having nonnegative fuzzy coefficients and nonnegative fuzzy variables,

(ii) FFLS having nonnegative fuzzy coefficients and unrestricted fuzzy variables,

(iii) FFLS having unrestricted fuzzy coefficients and nonnegative fuzzy variables,

(iv) FFLS having unrestricted fuzzy coefficients and unrestricted fuzzy variables,

(v) Fuzzy linear systems with crisp coefficient matrix and unrestricted fuzzy variables.

The numerical examples that are solved in Section 5 clearly demonstrate that the proposed method can solve FFLS with arbitrary coefficients as well as FFLS with nonnegative and other several types of restrictions. The proposed method thus fruitfully removes the constraints that are imposed by researchers and is therefore better to use in several engineering and scientific applications. However a future work still remains to evolve methods with improved computational complexity and to solve an arbitrary FFLS with nonlinear membership functions.

\section{References}

[1] L. A. Zadeh, "Fuzzy sets," Information and Control, vol. 8, no. 3, pp. 338-353, 1965.

[2] J. J. Buckley and Y. Qu, "Solving systems of linear fuzzy equations," Fuzzy Sets and Systems, vol. 43, no. 1, pp. 33-43, 1991.

[3] M. Friedman, M. Ming, and A. Kandel, "Fuzzy linear systems," Fuzzy Sets and Systems, vol. 96, no. 2, pp. 201-209, 1998.

[4] M. Friedman, M. Ming, and A. Kandel, "Duality in fuzzy linear systems," Fuzzy Sets and Systems, vol. 109, no. 1, pp. 55-58, 2000.

[5] T. Allahviranloo, "Numerical methods for fuzzy system of linear equations," Applied Mathematics and Computation, vol. 155, no. 2, pp. 493-502, 2004.

[6] T. Allahviranloo, "Successive overrelaxation iterative method for fuzzy system of linear equations," Applied Mathematics and Computation, vol. 162, pp. 189-196, 2004.

[7] T. Allahviranloo, "The adomian decomposition method for fuzzy system of linear equations," Applied Mathematics and Computation, vol. 163, no. 2, pp. 553-563, 2005.

[8] S. Abbasbandy, R. Ezzati, and A. Jafarian, "LU decomposition method for solving fuzzy system of linear equations," Applied Mathematics and Computation, vol. 172, no. 1, pp. 633-643, 2006.

[9] S. Abbasbandy, A. Jafarian, and R. Ezzati, "Conjugate gradient method for fuzzy symmetric positive-definite xsystem of linear equations," Applied Mathematics and Computation, vol. 171, no. 2, pp. 1184-1191, 2005.

[10] S. Abbasbandy and A. Jafarian, "Steepest descent method for system of fuzzy linear equations," Applied Mathematics and Computation, vol. 175, no. 1, pp. 823-833, 2006.

[11] M. Dehghan and B. Hashemi, "Iterative solution of fuzzy linear systems," Applied Mathematics and Computation, vol. 175, no. 1, pp. 645-674, 2006.
[12] M. Dehghan and B. Hashemi, "Solution of the fully fuzzy linear systems using the decomposition procedure," Applied Mathematics and Computation, vol. 182, no. 2, pp. 1568-1580, 2006.

[13] M. Dehghan, B. Hashemi, and M. Ghatee, "Solution of the fully fuzzy linear systems using iterative techniques," Chaos, Solitons and Fractals, vol. 34, no. 2, pp. 316-336, 2007.

[14] M. Dehghan, B. Hashemi, and M. Ghatee, "Computational methods for solving fully fuzzy linear systems," Applied Mathematics and Computation, vol. 179, no. 1, pp. 328-343, 2006.

[15] S. Muzzioli and H. Reynaerts, "Fuzzy linear systems of the form $A_{1} x+b_{1}=A_{2} x+b_{2}$," Fuzzy Sets and Systems, vol. 157, no. 7, pp. 939-951, 2006.

[16] M. Mosleh, S. Abbasbandy, and M. Otadi, "Full fuzzy linear systems of the form $A x+b=C x+d$," in Proceedings of the 1st Joint Congress on Fuzzy and Intelligent Systems, 2007.

[17] S. H. Nasseri, M. Sohrabi, and E. Ardil, "Solving fully fuzzy linear systems by use of a certain decomposition of the coefficient matrix," International Journal of Computational and Mathematical Sciences, vol. 2, pp. 140-142, 2008.

[18] T. Allahviranloo, N. Mikarilvand, N. A. Kiani, and R. H. Shabestari, "Signed decomposition of fully fuzzy linear systems," Application and Applied Mathematics, vol. 3, pp. 77-88, 2008.

[19] J. Gao, "A unified iterative scheme for solving fully fuzzy linear system," in Proceedings of the Global Congress on Intelligent Systems (GCIS '09), vol. 1, pp. 431-435, 2009.

[20] S. H. Nasseri, M. Matinfar, and Z. Kheiri, "Greville's method for the fully fuzzy linear system of equations," Advances in Fuzzy Sets and Systems, vol. 4, pp. 301-311, 2009.

[21] M. Mosleh, M. Otadi, and A. Khanmirzaie, "Decomposition method for solving fully fuzzy linear systems," Iranian Journal of Optimization, vol. 1, pp. 188-198, 2009.

[22] S. H. Nasseri and F. Zahmatkesh, "Huang method for solving fully fuzzy linear system of equations," The Journal of Mathematics and Computer Science, vol. 1, pp. 1-5, 2010.

[23] H. K. Liu, "On the solution of fully fuzzy linear systems," International Journal of Computational and Mathematical Sciences, vol. 6, pp. 29-33, 2010.

[24] S. H. Nasseri and M. Sohrabi, "Gram-schmidt approach for linear system of equations with fuzzy parameters," The Journal of Mathematics and Computer Science, vol. 1, pp. 80-89, 2010.

[25] A. Kumar, Neetu, and A. Bansal, "A new method to solve fully fuzzy linear system with trapezoidal fuzzy numbers," Canadian Journal on Science and Engineering Mathematics, vol. 1, pp. 45-56, 2010.

[26] T. Allahviranloo and M. Ghanbari, "Solving fuzzy linear system by homotopy perturbation method," International Journal of Computational Cognition, vol. 8, pp. 24-30, 2010.

[27] M. Mosleh and M. Otadi, "Regular splitting method for approximating linear system of fuzzy equations," Interntional Journal of Contemporary Mathematical Sciences, vol. 5, pp. 263-274, 2010.

[28] X. Sun and S. Guo, "Solution to general fuzzy linear system and its necessary and sufficient condition," Fuzzy Information and Engineering, vol. 3, pp. 317-327, 2009.

[29] J. F. Yin and K. Wang, "Splitting iterative methods for fuzzy system of linear equations," Computational Mathematics and Modeling, vol. 20, no. 3, pp. 326-335, 2009.

[30] D. Dubois and H. Prade, Fuzzy Sets and Systems: Theory and Applications, Academic Press, New York, NY, USA, 1980.

[31] A. Kaufmann and M. M. Gupta, Introduction to Fuzzy Arithmetic: Theory and Applications, Van Nostrand Reinhold, New York, NY, USA, 1991. 

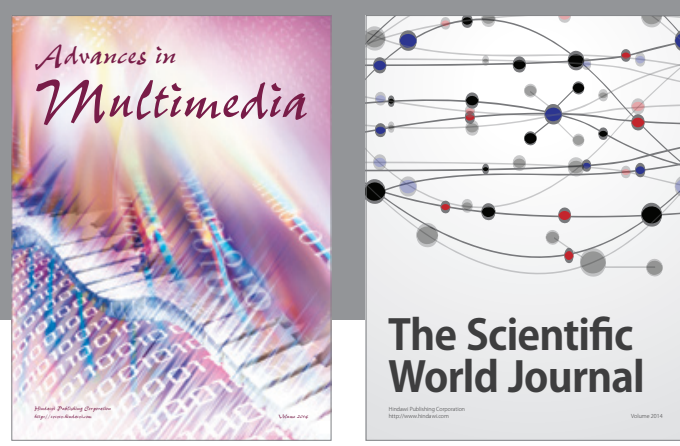

The Scientific World Journal
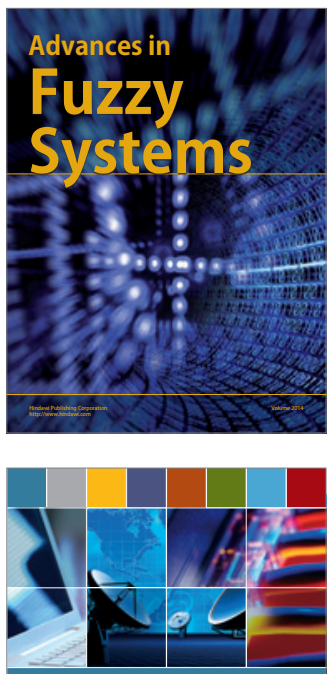

Computer Networks and Communications
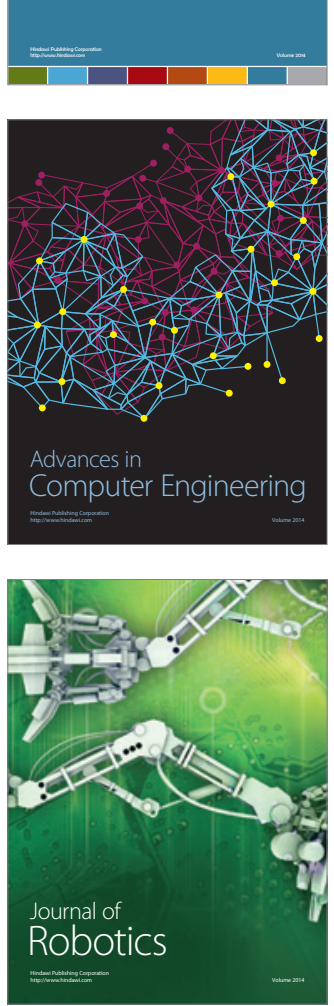
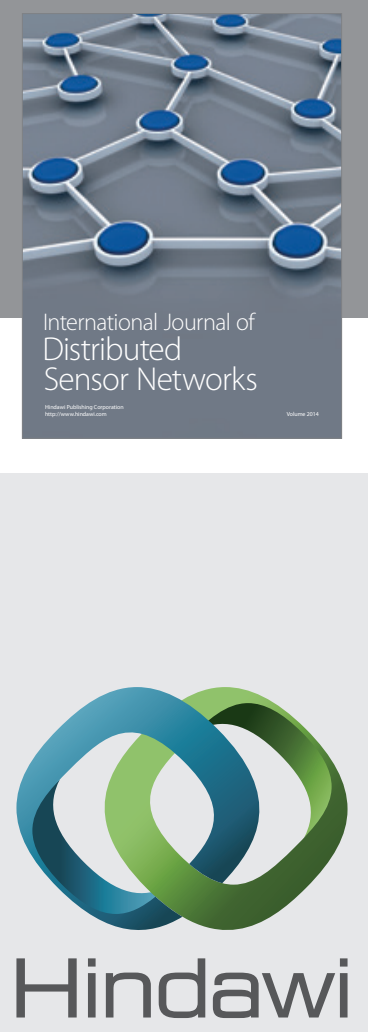

Submit your manuscripts at

http://www.hindawi.com
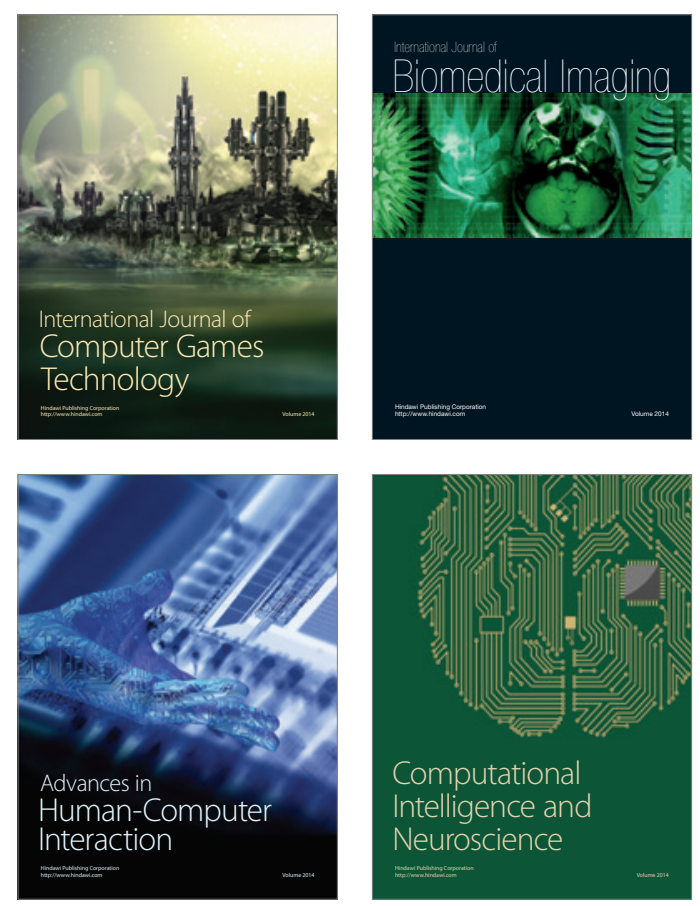
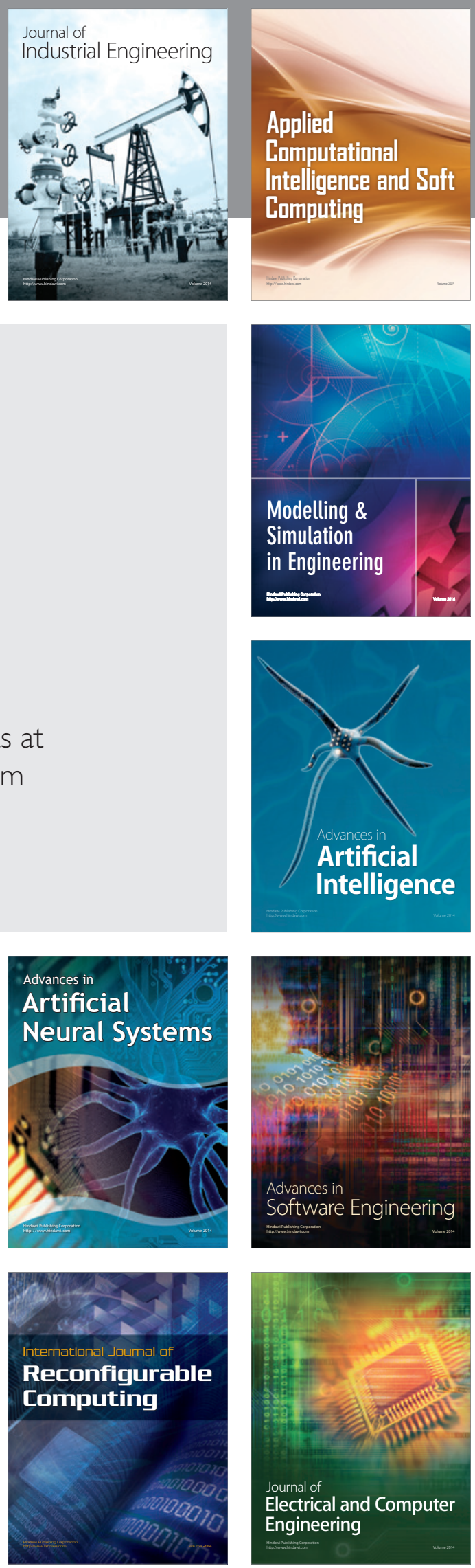